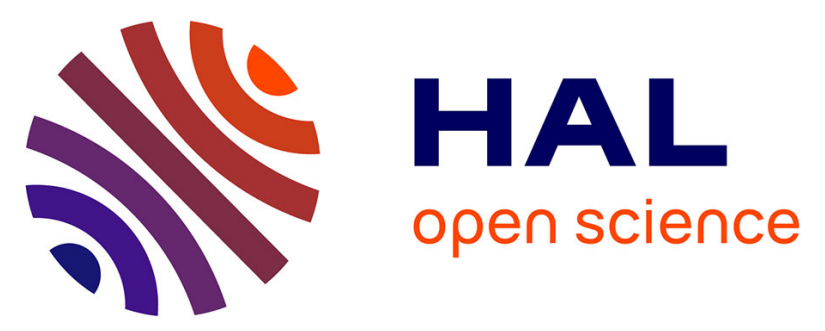

\title{
An application of quasi Monte Carlo methods for the numerical assessment of maintenance strategies
}

Jeanne Demgne, William Lair, Jérome Lonchampt, Sophie Mercier

\section{To cite this version:}

Jeanne Demgne, William Lair, Jérome Lonchampt, Sophie Mercier. An application of quasi Monte Carlo methods for the numerical assessment of maintenance strategies. 25th European Safety and Reliability Conference, ESREL 2015, 2015, Zurich, Switzerland. pp.907-916, 10.1201/b19094-122 . hal-01355100

\author{
HAL Id: hal-01355100 \\ https://hal.science/hal-01355100
}

Submitted on 22 Aug 2016

HAL is a multi-disciplinary open access archive for the deposit and dissemination of scientific research documents, whether they are published or not. The documents may come from teaching and research institutions in France or abroad, or from public or private research centers.
L'archive ouverte pluridisciplinaire HAL, est destinée au dépôt et à la diffusion de documents scientifiques de niveau recherche, publiés ou non, émanant des établissements d'enseignement et de recherche français ou étrangers, des laboratoires publics ou privés. 


\title{
An application of Quasi Monte Carlo methods for the numerical assessment of maintenance strategies
}

\author{
J. Demgne, W. Lair \& J. Lonchampt \\ EDF R\&D - Industrial Risk Management Department \\ Chatou - France
}

\author{
S. Mercier \\ Laboratoire de Mathématiques et de leurs Applications - Pau (UMR CNRS 5142) \\ Université de Pau et des Pays de l'Adour - France
}

\begin{abstract}
To improve the management of maintenance planning and spare parts ordering of a fleet of components, different investments plans need to be compared. A new investments plan is compared with a reference one through an economic variable called the Net Present Value (NPV). Classically, Monte Carlo simulations are used to assess economic indicators such as the expected NPV and the probability for the NPV to be negative which stands for the probability to regret the performed investments plan. In this document, we propose to use quasi Monte Carlo methods as an alternative to the Monte Carlo (MC) method, which replace the random uniform numbers of the MC method by deterministic sequences with better uniformity properties.
\end{abstract}

\section{INTRODUCTION}

In order to optimize the maintenance cost of a fleet of components, several investments plans are usually investigated. In our context, a fleet of identical components is considered. These components are expensive and their unavailability is also costly. Moreover, the manufacturing time of the components is long and consequently, the ordering process must be rigorous. One goal of asset management is to help deciding whether a new preventive maintenance strategy is better than another one. With that aim, the new investments plan is compared with a reference one, usually the one without planned investment, through an economic indicator called the Net Present Value (NPV). The NPV stands for the difference between the cumulated discounted cash-flows of both current and new investments plans. As failure dates of components are random, the NPV is also random. A first indicator for the assessment of an investment plan is the expected NPV. However, this quantity does not catch neither the NPV variability nor the probability for the NPV to be negative, which is a valuable quantity for decision making because it is the probability to regret the new investments plan. Figure 1 shows two probability distribution functions of the NPV (NPV 1 and NPV 2). In this figure, the expected NPV for strategy 2 is higher than for strategy 1 , but strategy 2 has the highest probability for the NPV to be negative. According to the risk aversion of the decision maker, strategy 2 is not necessarily better than strategy 1 . The NPV is hence an important variable to choose a relevant investments plan and its numerical assessment needs to be accurate. Of course, others indicators can be used to compare maintenance strategies. For example, the expected maintenance cost which is the most common economic indicator. Nevertheless, we will focus on the NPV which is used in the context of our study.

In order to model the random behaviour of the fleet of components, a Piecewise Deterministic Markov Process (PDMP) is used. PDMPs have been introduced by Davis (1984). Several methods have been used for the numerical assessment of a PDMP. Classically, Monte Carlo (MC) simulations are used to assess quantities of interest for a PDMP (Zhang et al. (2009)). The main drawback of MC method is its slow convergence. Thus, MC simulations require a long computational time to obtain accurate results. This may be prohibitive within an optimization algorithm. It is also possible to assess quantities of interest for a PDMP by finite volume methods (Eymard et al. (2008)) and quantization method (Brandejsky et al. (2012)).

In this article, quasi Monte Carlo (QMC) methods are proposed for the numerical assessment of the NPV. They are well-known for often having a faster convergence than MC method. These methods sub- 
stitute random uniform variables on $[0,1]^{d}, d \geq 1$ by deterministic sequences which have better uniformity properties. The uniformity of a sequence is measured by the so-called discrepancy (Lemieux (2009)). Sequences which have such good properties of uniformity are called Low Discrepancy Sequences (LDS). There are two families of LDS (Lemieux (2009)): lattices and digital nets/sequences. Two different QMC methods are proposed for the assessment of the NPV. The first QMC method consists in estimating the quantities of interest (expected NPV, probability for the NPV to be negative...) as an integral of a function. The dimension of LDS is then linked to the number of events occurring during the lifetime of the fleet. The second QMC method simulates several copies of a Markov chain in parallel and reorders copies at each step in ascending order. The sorting step allows to introduce a dependence between them and to provide a better estimation of the distribution of the Markov chain (Lécot and Tuffin (2004), Haddad et al. (2010)). In L'Ecuyer et al. (2008), the authors introduce the term "Array" to illustrate the parallel simulation of Markov chains and we follow this terminology in this paper. Randomized versions of the QMC methods are also used and consist in replacing the deterministic LDS of the QMC methods by randomized versions. An other randomized quasi Monte Carlo, called array randomized quasi Monte Carlo (ARQMC) method, is also presented (more details in L'Ecuyer et al. (2008)). As the LDS have better uniformity than random sequences, the QMC methods are theoretically more effective than the MC method. Thus, within an optimization algorithm, they will provide accurate results with a shorter computational time than the MC method. The aim of this paper is to apply and test the efficiency of the QMC method for the numerical assessment of maintenance strategies.

The structure of the article is as follows: Section 2 introduces both corrective and preventive maintenance strategies, and the NPV. Section 3 presents the constitutive variables of the PDMP which models the evolution of the fleet of components under maintenance strategies. Different methods for the numerical assessment of the NPV (MC, QMC and randomized QMC methods) are presented in Section 4. Numerical results are provided in Section 5 and a conclusion ends the paper in Section 6.

\section{PRESENTATION OF THE PROBLEM}

A fleet of $n$ identical and independent components is considered, which share a common stock of spare parts. These components are subject to both corrective and preventive replacements (CR and PR). At initial time, the stock is assumed to contain one single spare part and the supply time is deterministic, denoted by $\tau$. Moreover, the components of the fleet are assumed to be new at time $t=0$. The corrective and preventive maintenance strategies are compared through the

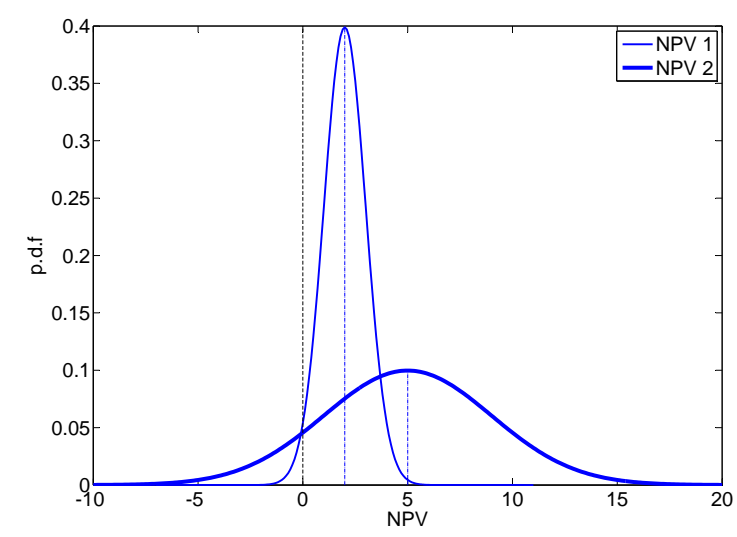

Figure 1: Comparison of probability distribution function (p.d.f) of two NPVs

NPV on a fixed operation horizon term denoted by $H$. As all components are new at the initial time, they have the same operating time $H$.

\subsection{The corrective replacement policy}

The corrective maintenance (CM) strategy consists in replacing a failed component by a new one if a spare part is available. If the stock is empty at a failure time, the failed component becomes unavailable and it remains down until a spare becomes available. The ordering process of a new spare part is as follows:

- On $[0, H-\tau[$, a new spare part is ordered at each failure of a component.

- On $[H-\tau, H]$, no order is made. Indeed, the spare part would be delivered after the operation horizon term.

\subsection{The preventive replacement policy}

Let $\zeta$ be a fixed deterministic time such that $\zeta<H-\tau$. The preventive maintenance (PM) strategy consists in preventively replacing at time $\zeta$ all components which did not fail before. The PM strategy is an exceptional maintenance. It involves significant investments and takes place only once on the operation term. With that aim, spare parts are ordered at time $\zeta-\tau$. The stock of spare parts is common to $\mathrm{CR}$ and PR. A CR has a priority on a preventive one. If $\mathrm{CR}$ are pending at time of the PM then, they are first carried out. After all pending $\mathrm{CR}$ have been performed at $\zeta, \mathrm{PM}$ actions take place if remaining spare parts are still available. When only a part of components awaiting for PR can be replaced (by lack of spare parts), the components to be replaced are randomly selected among all the equally likely components awaiting for PR. In this case, the PR of the remaining components is deferred until a new spare part becomes available.

The ordering process of a new spare part is the following: 
- On $[0, \zeta-\tau[$, a new spare part is ordered at each failure of a component.

- At time $\zeta-\tau$, if there are components that have never failed, then spare parts are ordered in readiness for their PR at time $\zeta$.

- On $] \zeta-\tau, \zeta[$, a spare part is still ordered at failure of a component $C$ (say) but only in the case where component $\mathrm{C}$ has already suffered a failure before. Indeed, in the opposite case, the PR of component $\mathrm{C}$ has been planned at time $\zeta$ and a spare part has already been ordered for its replacement. This spare part will be used for the $\mathrm{CR}$ of component $\mathrm{C}$ at $\zeta$.

- At time $\zeta$, spare parts arrive, which are used first for CR and next for PR. Note that some of these replacements may be deferred by lack of enough available spare parts.

- On $] \zeta, H-\tau$ [, a spare part is ordered at failure of a component $\mathrm{C}$ which has already been replaced (CR or PR). Indeed, in the other case, the PR of component $\mathrm{C}$ has been deferred at time $\zeta$ and an order is already in progress for its replacement.

- On $[H-\tau, H]$, no order is made.

The final goal is to optimize the time $\zeta$ where the components will be replaced. The optimal time will be the one which maximizes the expected NPV under the constraint that the probability to regret the investments is lower than a fixed threshold.

\subsection{Costs data}

The cost function associated to an investments plan takes into account costs due to $\mathrm{CR}$ or PR, purchase of spare parts and components unavailability. Discounted costs are considered. The discount rate is defined in John et al. (2009) as "the interest rate at which future receipts or payment are discounted to find their present value." In this paper, an exponential discounting is considered. Thus, if $C$ is a cost at time $t$, then the discounted cost is $C \times e^{-\alpha t}$ at time 0 with $\alpha$ the discount rate. The costs of CR and PR actions are respectively denoted $c_{r}$ and $c_{p}$. They are cashed at the replacement time. These costs do not include the cost of spare parts orders. The price of one spare part is denoted by $c_{A}$. It is cashed at delivery in case of a planned replacement at time $\zeta$. In the opposite case (any other time), it is cashed at time of order. The downtime cost of one component is denoted $C_{\text {ind }}$ and it is cashed at the end of the period of unavailability.

\subsection{The Net Present Value}

In John et al. (2009), the Net Present Value (NPV) is "the present value of a security or an investment project, found by discounting all present and future receipts and outgoings at an appropriate rate of discount. If the NPV calculated is positive, it is worthwhile investing in a project". Then, a positive NPV reflects the fact that the cash inflows deriving from an investment is greater than the cost of investment. The NPV can hence be seen as a relevant indicator for knowing whether an investment should be profitable or not.

As mentioned previously, the NPV stands for the difference between the cumulated discounted cashflows of both strategies (CM and PM). The NPV is zero as long as the two strategies coincide. The time $T$ when the two strategies first differ depends on the scenario: if all components have already suffered a failure before $\zeta-\tau$, then no PR is planned at time $\zeta$, and $T$ is equal to $H(N P V=0)$. On the contrary, if there is at least one component which has not suffered any failure up to time $\zeta-\tau$, then a spare part is ordered in view of its PR at time $\zeta$, and this makes the two strategies differ from time $T=\zeta-\tau$. Note that, even in that case, the two strategies coincide on $[0, \zeta-\tau[$ and the NPV is the difference of costs on $[\zeta-\tau, H]$. After time $\zeta-\tau$, the two maintenance strategies are assumed to evolve independently. However, they are correlated through their common history up to $\zeta-\tau$. Hence, the NPV is the difference of two dependent costs.

Assessing the NPV requires the joint modelling of the evolution of the components lifetimes, of the size of the stock, of the arrival times of the spare parts and of the cost functions under both maintenance strategies. This includes both discrete and continuous random parts. Piecewise Deterministic Markov Processes (PDMPs) are consequently well adapted.

\section{MODELLING THROUGH PDMPS}

\subsection{Description of parts of PDMP}

From a general point of view, Piecewise Deterministic Markov Processes have been introduced by Davis (Davis (1984)). A PDMP is a hybrid process $\left(I_{t}, X_{t}\right)_{t>0}$. The first part $I_{t}$ is discrete, with values in a discrete state space $E$. The second part $X_{t}$ represents a continuous variable which takes range in a Borel subset $B \subset \mathbb{R}^{d}$.

In our context, the following variables are the constitutive parts of our PDMP:

- $X_{t}=\left(X_{1, t}, \ldots, X_{n, t}\right)$ where the signification of $X_{j, t}$ differs according to whether the corresponding component is up or down. If it is up, $X_{j, t}$ is the predicted time for its future failure $\left(X_{j, t}>t\right)$. If it is down, $X_{j, t}$ is its last time of failure $\left(X_{j, t}<\right.$ $t$ ).

- $I_{t}=\left(I_{1, t}, \ldots, I_{n, t}\right)$ is failure indicators: for $1 \leq$ $j \leq n$, if the component corresponding to $X_{j, t}$ has never failed before $t$ then $I_{j, t}=1$, else $I_{j, t}=$ 0 . Thus at time $t, K_{t}=\sum_{j=1}^{n} I_{j, t}$ represents the 
number of components that have never been replaced on $[0, t]$.

- $S_{t}$ represents the number of available spare parts at time $t$.

- $D_{t}=\left(D_{1, t}, \ldots, D_{m, t}\right)$ represent the predicted times for spare parts arrivals.

- $L_{t}$ is the number of components at time $t$ awaiting for a deferred PR.

- $C_{t}$ is the cumulated discounted cost at time $t$.

The process $\left(Z_{t}\right)_{t \geq 0}=\left(Y_{t}, t\right)_{t \geq 0}=$ $\left(\left(X_{t}, I_{t}\right), S_{t}, D_{t}, L_{t}, C_{t}, t\right)_{t>0}$ is a PDMP whose discrete part is $\left(I_{t}, S_{t}, L_{t}\right)$ and continuous part is $\left(X_{t}, D_{t}, C_{t}, t\right)$. The part $\left(X_{t}, D_{t}, C_{t}\right)$ is constant between two jumps of the process $\left(Z_{t}\right)_{t>0}$. As a consequence, if $\left(T_{k}\right)_{k>0}$ represents the jump times of $\left(Z_{t}\right)_{t \geq 0}$, we have:

$Y_{t}=Y_{T_{k}}$ for $T_{k} \leq t<T_{k+1}$.

We also set $\left(Z_{k}\right)_{k \geq 0}=\left(Y_{T_{k}}, T_{k}\right)_{k \geq 0}$ to stand for the underlying Markov chain of the PDMP $\left(Z_{t}\right)_{t>0}$.

We add a superscript $(i)$ to all the previous notations and, CM and PM strategies are modelled with $\left(Z_{t}^{(1)}\right)_{t \geq 0}$ and $\left(Z_{t}^{(2)}\right)_{t \geq 0}$, respectively.

We do not provide here the details of the transitions of $\left(Z_{t}^{(i)}\right)_{t \geq 0}, i=\{1,2\}$, due to the reduced size of the paper.

\subsection{The Net Present Value}

The NPV stands for the difference between the cumulated discounted costs of both CM and PM strategies. The NPV is defined as follows:

$$
\begin{aligned}
& N P V(H)=N P V([0, H]) \\
& =N P V([0, \zeta-\tau[)+N P V([\zeta-\tau, H]) \\
& =N P V([\zeta-\tau, H]) \mathbf{1}_{\left\{K_{\zeta-\tau}>0\right\}} \\
& =\left(C^{(1)}([\zeta-\tau, H])-C^{(2)}([\zeta-\tau, H])\right) \mathbf{1}_{\left\{K_{\zeta-\tau}>0\right\}}
\end{aligned}
$$

where $C^{(1)}([\zeta-\tau, H])$ and $C^{(2)}([\zeta-\tau, H])$ are dependent through the state of components and stock at time $(\zeta-\tau)^{-}$.

The quantities of interest are $\mathbb{E}[N P V(H)]$, $\mathbb{P}(N P V(H) \leq u)$ where $u \in \mathbb{R}$. These quantities depend on all events which occur during $[0, H]$ on both strategies. They can be written as $\mathbb{E}\left[\Phi\left(\left(Z_{k_{1}}^{(1)}, Z_{k_{2}}^{(2)}\right)_{0 \leq k_{1} \leq N_{H}^{(1)}, 0 \leq k_{2} \leq N_{H-\zeta+\tau}^{(2)}}\right)\right]$ where $N_{H}^{(1)}$ (resp. $N_{H-\zeta+\tau}^{(2)}$ ) stands for the number of jumps of $Z^{(1)}$ (resp. $Z^{(2)}$ ) on $[0, H]$ (resp. $[\zeta-\tau, H]$ ).

\section{NUMERICAL ASSESSMENT OF THE NET PRESENT VALUE}

\subsection{Monte Carlo method}

Sample paths of each Markov chain $\left(Z_{k}^{(i)}\right)_{k \geq 0}, i=$ $\{1,2\}$, are sequentially simulated and a sample of $N P V(H)$ is derived using Equation (2). Next, the expected $N P V(H)$ and the cumulated distribution function (c.d.f.) of $N P V(H)$ are estimated by empirical mean and empirical c.d.f., respectively.

Confidence intervals for these estimations are given by the central limit theorem. Quasi Monte Carlo methods are now introduced.

\subsection{Quasi Monte Carlo method}

As told in the introduction, the QMC method estimates the quantities of interest for the NPV as an integral of a function. In Subsection 3.2, we have seen that all quantities of interest can be written as a function of $N_{H}=N_{H}^{(1)}+N_{H}^{(2)}$ random variables. Then, $N_{H}$ also is a random variable. However, the dimension of the LDS must be known in advance and therefore it can not be a random variable. To overcome this, $M \in \mathbb{N}^{*}$ is chosen such that $\mathbb{P}\left(M>N_{H}\right)>1-\varepsilon$, with $\varepsilon$ very small and a LDS with dimension $M$ is built. Just as for the MC method, sample paths of Markov chain modelling each maintenance strategy are sequentially simulated using the $M$-dimensional LDS. A sample of $N P V(H)$ is then derived.

As presented in the introduction and following Krykova (2003), we use here a digital net: the Sobol sequence. In Figure 2, a sample of 64 realizations of random uniform distribution on $[0,1]^{2}$ and 64 points of Sobol sequence in dimension 2 are compared. This figure illustrates the better uniformity of a Sobol sequence than a uniform random sample, for which many areas are left unexplored.

QMC method is known to lose its effectiveness when the dimension of the LDS becomes large. Here, the dimension of LDS can become large when the number of components increases. To overcome this limit, we propose to use the Array Quasi Monte Carlo method.

\subsection{Array Quasi Monte Carlo method}

As said in the introduction, the Array Quasi Monte Carlo (AQMC) method consists in simulating several copies of a Markov chain in parallel using elements of LDS and reordering copies at each step ascending order. Unlike the QMC method where an element of the LDS is used to simulate one path of the Markov chain, the AQMC method uses one element of the LDS to assess one step of the path. The dimension of the LDS is not linked to the number of jumps of the Markov chain but is equal to the number of uniform random 

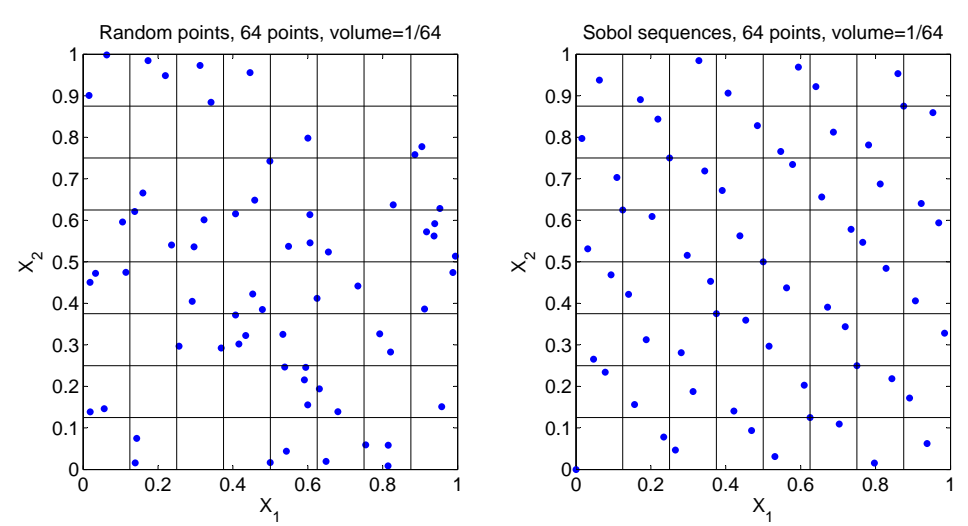

Figure 2: Comparison of random uniform variable (left) and Sobol sequence (right)

variables required to simulate one transition of the Markov chain. The AQMC method hence allows to reduce significantly the dimension of the LDS when compared to the QMC method. Moreover, the dimension of the LDS is not random any more like for the QMC method but it is deterministic.

In order to assess the $N P V(H)$, the AQMC method simulates $N$ copies of the Markov chain in parallel for each maintenance strategy and reorders them at each step. Remember that both maintenance strategies are dependent through their common history up to $\zeta-\tau$. Thus, during the sorting step of copies of each Markov chain, it is primordial to keep the dependence between copies which have the same history. Here, two LDS are considered. The first LDS assesses the first times of future failure for all components at the initial time. This permits to have a good repartition for the initial copies of Markov chains. The second LDS is used to evaluate the transitions of Markov chains. A sample of $N P V(H)$ is then calculated using a sample path of Markov chains.

Unlike the MC method where confidence intervals of the estimations can be constructed using the central limit theorem, Quasi Monte Carlo methods are deterministic and they do not provide the error of the estimations. However, through the Koksma-Hlawka theorem (Lemieux 2009), an upper bound of the error of estimation by Quasi Monte Carlo methods can be assessed. In practice, this upper bound is difficult to calculate and overestimates the error. Other methods which allow to construct confidence intervals of the estimations are now presented.

\subsection{Randomized (Array) Quasi Monte Carlo methods}

As told in the introduction, randomized Quasi Monte Carlo methods use randomized LDS. A randomized version of LDS is a random sequence which has the two following properties (Lemieux (2009)):

1. each randomized point is uniformly distributed on $[0,1]^{d}, d \geq 1$,

2. the regularity of the points is preserved (in the sense of low discrepancy).
Thus, randomized Quasi Monte Carlo methods have advantages over both Monte Carlo method (confidence intervals) and Quasi Monte Carlo methods (better regularity than a random sample). Several randomization methods presented in Lemieux (2009) are available. In this paper, the random shift method is used.

To assess $N P V(H)$ with randomized Quasi Monte Carlo methods, the randomized LDS replaces deterministic LDS in each deterministic methods. Thus, in the Randomized Quasi Monte Carlo (RQMC) method, a $M$-dimension randomized LDS is considered and in the Randomized Array Quasi Monte Carlo (RAQMC) method, two LDSs are considered. In the Array Randomized Quasi Monte Carlo (ARQMC) method, presented in (L'Ecuyer et al. (2008)), two LDS also are considered and several copies are simulated in parallel and reordered at each step. In the ARQMC method, the LDS for assessing one transition of Markov chains is randomized at each step while in the RAQMC method it is randomized only once at the beginning.

Let be $\theta=\mathbb{E}[h(N P V(H))], h$ a function. To estimate $\theta$ with randomized Quasi Monte Carlo methods, a sample of estimations $\left\{\hat{\theta}_{1}, \ldots, \hat{\theta}_{J}\right\}$ is obtained using $J$ independent randomizations of LDS with deterministic methods. Then, the estimation of $\theta$ is given by

$\bar{\theta}=\frac{1}{J} \sum_{j=1}^{J} \hat{\theta}_{j}$

Confidence interval of $\bar{\theta}$ is given by using the central limit theorem on the $J$ independent estimations of $\theta$. Thus Randomized Quasi Monte Carlo methods depend on two parameters: the number of elements of LDS and the number of independent randomizations.

\section{NUMERICAL RESULTS ON A FICTITIOUS CASE STUDY}

\section{$5.1 \quad$ Numerical parameters}

To assess $\mathbb{E}[N P V(H)]$ and $\mathbb{P}(N P V(H) \leq 0)$, the following parameters are considered: 
- Operation horizon time $H=60$;

- Supply time $\tau=1$;

- Predicted time to preventive maintenance actions $\zeta=43$

- Probability distribution of components time to failure: Weibull distribution $W(48 ; 2.6)$ with c.d.f.

$F(x)=\left(1-e^{-\left(\frac{x}{48}\right)^{2.6}}\right) \mathbf{1}_{\{x \geq 0\}}$

and $F(\zeta)=1-e^{-\left(\frac{43}{48}\right)^{2.6}} \simeq 0.5282$

$\mathbb{E}[X] \simeq 42.6341$ and $\operatorname{Var}(X) \simeq 310.2542$

- Continuous discount rate $\alpha=0.075$;

- Corrective replacement cost $c_{r}=200 €$;

- Preventive replacement cost $c_{p}=190 €$;

- Cost of downtime $C_{i n d}=58400 €$ per year;

- Purchasing price of one spare part $c_{A}=500 €$.

Here, the parameter $\zeta$ is fixed in order to illustrate the QMC methods.

\subsection{Comparison indicators of methods}

The different methods are compared through the following indicators.

\section{Relative error}

Let us denote by $\theta=\mathbb{E}[h(N P V(H))]$ where $\theta$ is either $\mathbb{E}[N P V(H])$ or $\mathbb{P}(N P V(H) \leq 0)$. $\hat{\theta}=\frac{1}{N} \sum_{i=1}^{N} h\left(n p v_{i}(H)\right)$ represents an empirical estimation of $\theta$ by deterministic methods where $\left(n p v_{i}(H)\right)_{1<i<N}$ is a sample of $N P V(H)$. The relative error is defined by:

$\varepsilon=\left|\frac{\hat{\theta}-\theta}{\theta}\right|$

For the random methods (MC, RQMC, RAQMC and ARQMC), the mean of the relative error on $J$ independent simulations of MC method or $J$ independent randomizations of LDS is evaluated. Let $\varepsilon_{j}=\left|\frac{\hat{\theta}_{j}-\theta}{\theta}\right|$ where $\hat{\theta}_{j}=\frac{1}{N} \sum_{i=1}^{N} h\left(n p v_{i}^{j}(H)\right)$ is an empirical estimation of $\theta$ obtained in the $j^{\text {th }}$ simulation of MC method or randomization of LDS. Then, the mean of the relative error is:

$\bar{\varepsilon}=\frac{1}{J} \sum_{j=1}^{J} \varepsilon_{j}$

In the numerical assessment, we set:
- $\varepsilon_{e x p}$ and $\bar{\varepsilon}_{\text {exp }}$ : the relative error and the mean of relative error on the expected $N P V(H)$, respectively;

- $\varepsilon_{\text {proba }}$ and $\bar{\varepsilon}_{\text {proba }}$ : the relative error and the mean of relative error on the probability for the $N P V$ to be negative at time $H$, respectively.

\section{Variance reduction factor}

In addition to the mean of relative error, random methods are also compared through their variance. The variance of different randomized Quasi Monte Carlo methods is compared with the variance of MC method through the variance reduction factor. For $J$ independent simulations or randomizations, the variance is defined by

$$
\operatorname{Var}\left(\frac{1}{J} \sum_{j=1}^{J} \hat{\theta}_{j}\right)=\frac{1}{J} \operatorname{Var}\left(\hat{\theta}_{1}\right)
$$

Then, the variance reduction factor, denoted by $V R F$, is given by

$$
V R F=\frac{\operatorname{Var}^{r Q M C}\left(\frac{1}{J} \sum_{j=1}^{J} \hat{\theta}_{j}\right)}{\operatorname{Var}^{M C}\left(\frac{1}{J} \sum_{j=1}^{J} \hat{\theta}_{j}\right)}=\frac{\operatorname{Var}^{r Q M C}\left(\hat{\theta}_{1}\right)}{\operatorname{Var}^{M C}\left(\hat{\theta}_{1}\right)}
$$

where $\mathrm{RQMC}$ can be RQMC, RAQMC or ARQMC. In the numerical assessment, $J=2^{9}=512$ independent simulations or randomizations are considered.

Remark 1 The reference values are obtained with MC method using $10^{9}$ independent simulations.

\subsection{Four components}

Table 1: Comparison of deterministic methods - 4 components

\begin{tabular}{ccccc}
\hline & Methods & CPU time(s) & $\varepsilon_{\text {exp }}$ & $\varepsilon_{\text {proba }}$ \\
\hline \multirow{2}{*}{$N=32768$} & $Q M C$ & 0.65 & $1.79 \times 10^{-3}$ & $4.43 \times 10^{-5}$ \\
& $A Q M C$ & 1.22 & $2.25 \times 10^{-2}$ & $2.19 \times 10^{-4}$ \\
\hline \multirow{2}{*}{$N=524288$} & $Q M C$ & 11.73 & $5 \times 10^{-4}$ & $1.04 \times 10^{-5}$ \\
& $A Q M C$ & 25.04 & $1.69 \times 10^{-3}$ & $2.37 \times 10^{-4}$ \\
\hline
\end{tabular}

Table 2: Comparison of random methods - 4 components

\begin{tabular}{ccccc}
\hline & Methods & CPU time(s) & $\varepsilon_{\text {exp }}$ & $\varepsilon_{\text {proba }}$ \\
\hline \multirow{5}{*}{$N=32768$} & $M C$ & 0.67 & 0.029 & 0.0017 \\
& $R Q M C$ & 0.95 & 0.016 & 0.0011 \\
& $R A Q M C$ & 1.5 & 0.0176 & 0.0011 \\
& $A R Q M C$ & 1.72 & 0.018 & 0.0012 \\
\hline \multirow{5}{*}{$N=524288$} & $M C$ & 12.12 & 0.0085 & $4.66 \times 10^{-4}$ \\
& $R Q M C$ & 13.52 & 0.0031 & $2.11 \times 10^{-4}$ \\
& $R A Q M C$ & 31.88 & 0.0041 & $2.74 \times 10^{-4}$ \\
& $A R Q M C$ & 40.4 & 0.0046 & $2.68 \times 10^{-4}$ \\
\hline
\end{tabular}

The reference values for $\mathbb{E}[N P V(60)]$ and $\mathbb{P}(N P V(60) \leq 0)$ are:

$\hat{\mu}_{r e f}=44.4508$ and $I C_{95 \%}\left(\hat{\mu}_{r e f}\right)=[44.4329,44.4687]$

$\hat{p}_{\text {ref }}=0.87279$ and $I C_{95 \%}\left(\hat{p}_{\text {ref }}\right)=[0.87277,0.87282]$ 

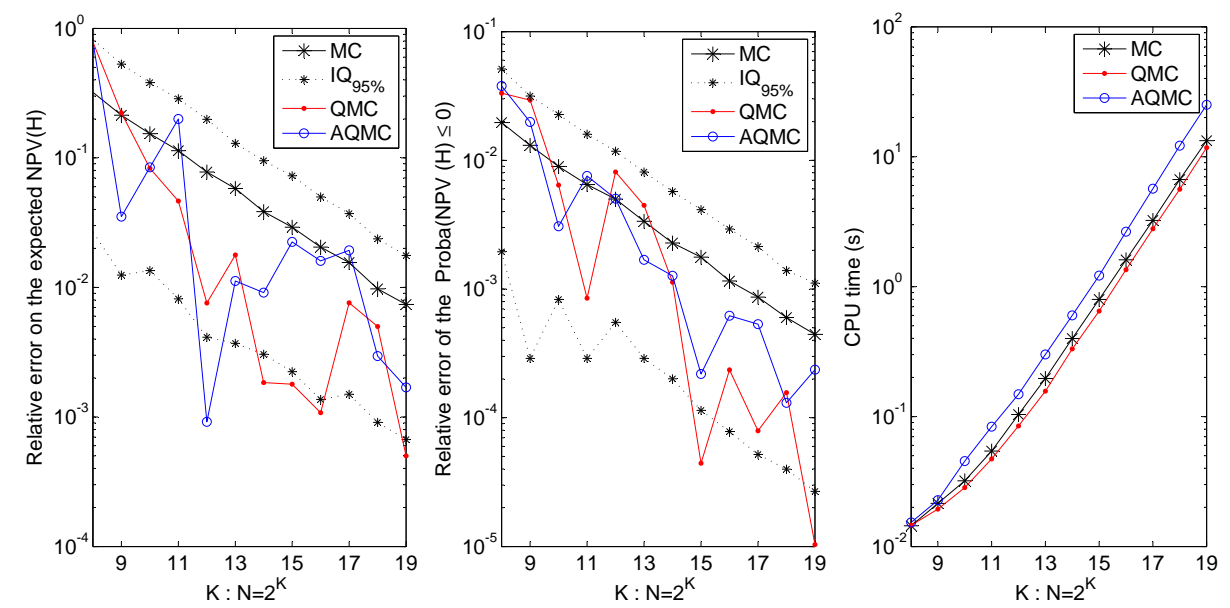

Figure 3: Relative error on $\hat{\mu}_{\text {ref }}$ (left), $\hat{p}_{\text {ref }}$ (center) and computational time (right) - 4 components

Table 3: Variance reduction factor - 4 components

\begin{tabular}{ccccc}
\hline & \multicolumn{2}{c}{$N=32768$} & \multicolumn{2}{c}{$N=524288$} \\
\cline { 2 - 5 } & $V R F_{\text {exp }}$ & $V R F_{\text {proba }}$ & $V R F_{\text {exp }}$ & $V R F_{\text {proba }}$ \\
\hline$R Q M C$ & 0.311 & 0.433 & 0.176 & 0.235 \\
$R A Q M C$ & 0.351 & 0.464 & 0.323 & 0.344 \\
$A R Q M C$ & 0.363 & 0.511 & 0.319 & 0.337 \\
\hline
\end{tabular}

$I C_{95 \%}(\cdot)$ represents a $95 \%$ confidence interval for the estimation. Figure 3 represents the evolution of relative errors and CPU time as a function of the number of points of the LDS for quasi Monte Carlo methods. For MC method, the mean of relative error, the $95 \%$ inter-quantile interval $\left(I Q_{95 \%}\right)$ for $J$ independent relative errors and the mean of CPU times are plotted. MC, QMC and AQMC have a good estimation of both indicators $\left(\hat{\mu}_{r e f}, \hat{p}_{r e f}\right)$ and their relative errors converge towards 0 . We can also see that for most values of $N$, the relative errors of deterministic methods are below the mean of relative errors of MC method. This reflects that on average, deterministic methods have a better estimation than MC method. Table 1 represents values of deterministic methods for $N=2^{15}=32768$ and $N=2^{19}=524288$. We can see that QMC method has relative errors and CPU time smaller than AQMC method. Thus, QMC method appears as the best deterministic method for the evaluation of $N P V(60)$ with four components. Table 2 compares the mean of relative errors and mean of CPU time for random methods for $N=2^{15}$ and $N=2^{19}$. We can see that different randomized quasi Monte Carlo methods are more accurate than MC method and have a variance smaller than the MC one $(V R F<1$, see Table 3$)$. Nevertheless, RAQMC and ARQMC have a long CPU time due to the sorting step of the Markov chains.

\subsection{Ten components}

The following reference values and confidence intervals are considered:

$\hat{\mu}_{r e f}=467.048$ and $I C_{95 \%}\left(\hat{\mu}_{r e f}\right)=[466.999,467.098]$

$\hat{p}_{\text {ref }}=0.47242$ and $I C_{95 \%}\left(\hat{p}_{\text {ref }}\right)=[0.47239,0.47246]$
Table 4: Comparison of deterministic methods - 10 components

\begin{tabular}{ccccc}
\hline & Methods & CPU time(s) & $\varepsilon_{\text {exp }}$ & $\varepsilon_{\text {proba }}$ \\
\hline \multirow{2}{*}{$N=65536$} & $Q M C$ & 5.08 & $5.56 \times 10^{-3}$ & $4.71 \times 10^{-3}$ \\
& $A Q M C$ & 6.86 & $2.85 \times 10^{-4}$ & $1.56 \times 10^{-3}$ \\
\hline \multirow{2}{*}{$N=262144$} & $Q M C$ & 19.1 & $1.3 \times 10^{-3}$ & $3.83 \times 10^{-3}$ \\
& $A Q M C$ & 29.49 & $8.45 \times 10^{-4}$ & $4.56 \times 10^{-4}$ \\
\hline
\end{tabular}

Table 5: Comparison of random methods - 4 components

\begin{tabular}{ccccc}
\hline & Methods & CPU time(s) & $\varepsilon_{\text {exp }}$ & $\varepsilon_{\text {proba }}$ \\
\hline \multirow{5}{*}{$N=65536$} & $M C$ & 4.11 & 0.0053 & 0.0035 \\
& $R Q M C$ & 5.37 & 0.0033 & 0.0024 \\
& $R A Q M C$ & 11.61 & 0.0034 & 0.0025 \\
& $A R Q M C$ & 12.4 & 0.0036 & 0.0026 \\
\hline \multirow{5}{*}{$N=262144$} & $M C$ & 18.04 & $2.57 \times 10^{-3}$ & $1.64 \times 10^{-3}$ \\
& $R Q M C$ & - & - & - \\
& $R A Q M C$ & 62.97 & $1.43 \times 10^{-3}$ & $1.15 \times 10^{-3}$ \\
& $A R Q M C$ & 67.36 & $1.45 \times 10^{-3}$ & $1.23 \times 10^{-3}$ \\
\hline
\end{tabular}

As in the case of four components, Figure 4 represents the evolution of the relative errors and the CPU time for the deterministic methods; the mean of relative errors and of CPU time and the 95\% interquantile interval $\left(I Q_{95 \%}\right)$ of relative errors for the $\mathrm{MC}$ method. We can see that all methods converge when $N$ increases. Table 4 shows that for $N=2^{16}=65536$ and $N=2^{18}=262144$ the AQMC method is more accurate but has a higher CPU time than QMC method. However, the results of AQMC methods with $N=2^{16}$ are obtained more quickly and are more accurate than the QMC ones with $N=2^{18}$. As Tables 2 and 3, Tables 5 and 6 show that randomized quasi Monte Carlo methods are more accurate and more stable than MC method. Note that the RQMC method is limited by memory problem to $N=2^{18}$ (on a standard laptop) whereas RAQMC and ARQMC methods can be used with higher $N$.

Table 6: Variance reduction factor - 10 components

\begin{tabular}{ccccc}
\hline & \multicolumn{2}{c}{$N=65536$} & \multicolumn{2}{c}{$N=262144$} \\
\cline { 2 - 5 } & $V R F_{\text {exp }}$ & $V R F_{\text {proba }}$ & $V R F_{\text {exp }}$ & $V R F_{\text {proba }}^{\prime}$ \\
\hline$R Q M C$ & 0.386 & 0.478 & - & - \\
$R A Q M C$ & 0.396 & 0.559 & 0.307 & 0.515 \\
$A R Q M C$ & 0.420 & 0.582 & 0.309 & 0.542 \\
\hline
\end{tabular}



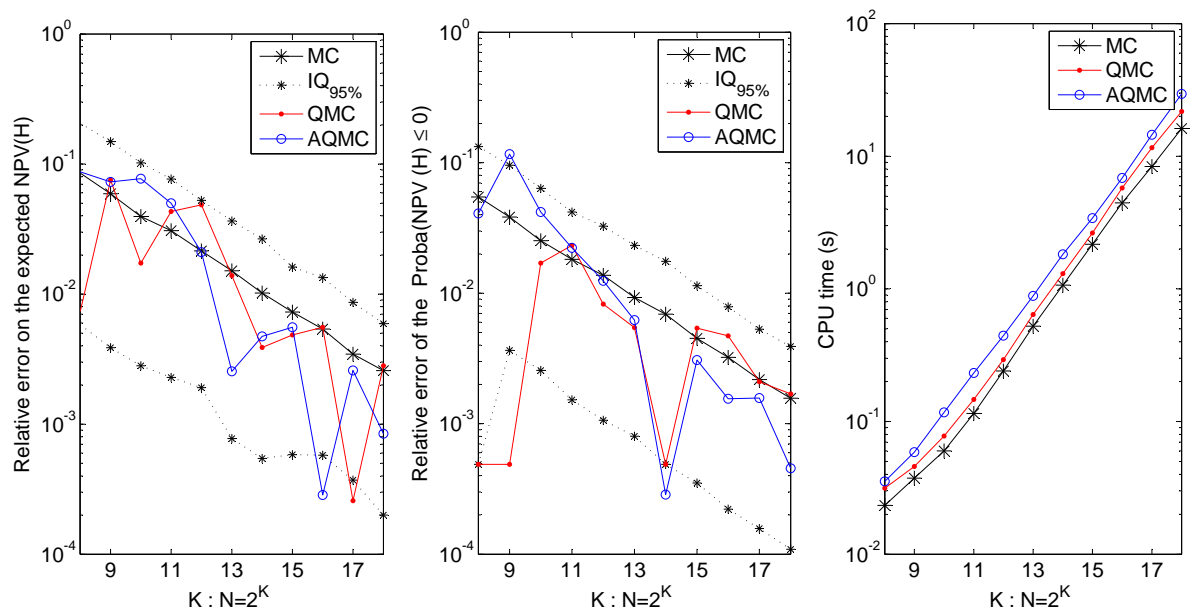

Figure 4: Relative error on $\hat{\mu}_{\text {ref }}$ (left), $\hat{p}_{\text {ref }}$ (center) and computational time (right) - 10 components

\section{CONCLUSION}

Two quasi Monte Carlo methods are proposed for the numerical assessment of complex PDMPs. In the QMC method, the dimension of the LDS is linked to the number of steps of the PDMPs and consequently, to the number of components as well. This is restricted to fleets with a few components because in the opposite case, a memory problem happens. In the AQMC method, the dimension of the LDS is equal to the number of random variables required for one single event. That significantly reduces the dimension of the LDS. Looking at the specific results, Tables 1 and 2 (four components) show that with an equivalent CPU time, the QMC method has a reduction factor on the relative errors greater than 10 when compared with the MC method. In Tables 4 and 5 (ten components), the MC and QMC methods provide similar results while the AQMC method is more accurate: for example, the relative error on the expected NPV of the AQMC method is equal to $2.849 \times 10^{-4}$ in 6.863 CPU time while the MC method has a relative error on the expected NPV equal to $2.569 \times 10^{-3}$ in 18.045 CPU time. For this example, the reduction factor on the relative error is around 10 in favour of the mostly thrice quicker AQMC method when compared to MC method. Based on these results (and on others not provided here), it seems that both QMC and AQMC methods are promising alternatives to the more standard MC methods for the numerical assessment of PDMPs. To be more specific, in our context, QMC method will be the most effective for a fleet of less than 10 components whereas the AQMC method will be the most efficient for a fleet of more than 10 components. The effectiveness of these methods should be greatly appreciated within a preventive maintenance optimization algorithm, which is our ultimate goal. It is important to highlight that all developments that have been presented in this paper can be generalized to any other assessment of two maintenance strategies. The case used in this communication is only given for the illustrative purposes and we can imagine that a black box MC simulator can be replaced by a black box QMC simulator for any industrial issue.

\section{REFERENCES}

Brandejsky, A., B. De Saporta, \& F. Dufour (2012). Numerical method for expectations of piecewise Deterministic Markov Processes. Communications in Applied Mathematics and Computational Science 7(1), 63-104.

Davis, M. H. A. (1984). Piecewise Deterministic Markov Processes: A general class of non diffusion stochastic models. Journal of the Royal Statistical Society. Series B (Methodological) 46(3), 353-388.

Eymard, R., S. Mercier, \& A. Prignet (2008). An implicit finite volume scheme for a scalar hyperbolic problem with measure data related to piecewise Deterministic Markov Processes. Journal of Computational and Applied Mathematics 222(2), 293-323.

Haddad, R. E., C. Lécot, P. L'Ecuyer, \& N. Nassif (2010). Quasi-Monte Carlo methods for Markov chains with continuous multidimensional state space. Mathematics and Computers in Simulation 81, 560-567.

John, B., H. Niga, \& M. Gareth (2009). A dictionary of economics (3rd ed.). Oxford : Oxford University Press.

Krykova, I. (2003, December). Evaluating of pathdependent securities with low discrepancy methods. Master's thesis, Worcester polytechnic institute.

Lécot, C. \& B. Tuffin (2004). Quasi Monte-Carlo methods for estimating transient measures of discrete time Markov chains. In Monte Carlo and Quasi Monte Carlo Methods 2002, pp. 329-343. Springer.

L'Ecuyer, P., C. Lécot, \& B. Tuffin (2008). A Randomized Quasi-Monte Carlo simulation method for Markov chains. Operations research 56(4), 958-975.

Lemieux, C. (2009). Monte Carlo and Quasi Monte-Carlo Sampling. Mathematics and Statistics. Springer-Verlag New York.

Zhang, H., F. Dufour, Y. Dutuit, \& K. Gonzalez (2009). Piecewise Deterministic Markov Processes and dynamic reliability. Journal of Risk and Reliability 222(4), $545-551$. 